\title{
Coherentism and the symmetry of epistemic support
}

\author{
Nicholas Shackel
}

In a recent paper Tom Stoneham (2007) offered a reductio of coherentism. In this paper I defend Stoneham from a criticism made by Darrell Rowbottom (2007) and I offer two proofs. The first is of an early step in Stoneham's argument, a step which is not (in my opinion) adequately argued by Stoneham; the second is that holistic coherentism is logically equivalent to the symmetry and quasi-transitivity ${ }^{1}$ of epistemic support and a condition on justified beliefs. I rebut Rowbottom partly because my proof uses the premiss he is criticising. I offer the proofs because I think the results are a matter of independent interest. For example, Thagard (2000) takes symmetry as an axiom, but perhaps he need not, or perhaps it, together with his view of explanation, commits him to holism. My proofs are independent of the criticisms of Stoneham made by Christoph Jäger (2007) and Paul Noordhof (2007).

Stoneham's first premiss is his characterization of coherentism:

(1) There are finite sets of beliefs such that each member of the set is epistemically supported by some other members (and nothing else). (Coherentism) (2007: 254)

\footnotetext{
${ }^{1}$ I stipulate that ' $L$ is quasi-transitive' means that $\forall x \forall y \forall z(x \neq z \wedge L x y \wedge L y z \rightarrow L x z)$.
} 
Later, Stoneham suggests that the negation of (1) is 'a pretty good definition of foundationalism' (2007: 257). Rowbottom thinks (1) cannot characterize coherentism because it can be shown to be true independently of coherentism. Rowbottom proposes a thought experiment in which a subject's memories are erased and replaced with three beliefs which apparently support one another (contents: 'there is a unicorn'; 'there is a white unicorn'; 'all unicorns are white'). Rowbottom's argument is essentially this:

(A) the brainwashed subject's beliefs are each epistemically supported by his two other beliefs and nothing else.

(B) A is true independently of whether foundationalism or coherentism is true.

(C) Therefore there are finite sets of beliefs such that each member of the set is epistemically supported by some other members (and nothing else). (A, Existential generalization)

(D) Therefore (1) is true independently of whether foundationalism or coherentism is true. $(\mathrm{A}, \mathrm{B}, \mathrm{C})$

(E) Therefore (1) does not characterize coherentism. (D)

So if Rowbottom is right, coherentism cannot be refuted by a reductio of (1).

\section{Rebutting Rowbottom}

Rowbottom appeals directly to our intuitive understanding of epistemic support. The claimed epistemic support among the trio of beliefs is constituted by relations between their contents and nothing else: two entailments and one instance of weak inductive support. ${ }^{2}$ This seems plausible, but the fact that it is controversial whether known entailment is sufficient for the transmission of warrant or knowledge ${ }^{3}$ shows that it cannot be assumed that epistemic relations follow entailments.

To advance on this I think we need to look carefully at the definition of epistemic support offered by Stoneham:

Epistemic support: 'A belief $\mathrm{b}(p)$ is epistemically supported by the belief $\mathrm{b}(q)$ iff $\mathrm{b}(p)$ 's justifiedness or reasonableness (for a subject at a time) consists in part in $\mathrm{b}(p)$ standing in some relation of dependence to $\mathrm{b}(q) \cdot{ }^{\prime}(2007: 254)$

${ }^{2}$ Rowbottom also mentions even simpler examples, such as $\{s, r, s \wedge r\}$, where the putative epistemic support is all entailment.

${ }^{3}$ For example, Dretske's (1970) example of having a visual warrant for believing that an animal is a zebra and knowing that if it is a zebra it is not a disguised mule whilst not having a warrant for believing that it is not a cleverly disguised mule (and hence not knowing that it is not). For a recent view on the controversy see Silins 2005. 
I think Stoneham is right to relate epistemic support to justification and dependence. ${ }^{4} \mathrm{~A}$ justified belief is one that depends in the right way on a sufficiency of epistemic support of the right sort. Rowbottom stipulates that the beliefs in his example 'are not justified ... except insofar as they might cohere' (2007: 345) but evidently does not think that that might cause any problems for the claimed epistemic support. I think that being persuaded by Rowbottom's case requires us to underplay the relations between epistemic support, justification and dependence.

Looking at the right-hand side of Stoneham's definition of epistemic support, we see a condition that is a conjunction: that $b(p)$ is justified and its justifiedness depends on $b(q)$. Under this definition, then, unjustified beliefs cannot be epistemically supported. That sounds wrong. There is a temptation to think that the epistemic support between a belief and an entailing belief can be sturdy even if the first belief is unjustified. On the other hand, it also sounds wrong to say that a belief lacking any justification nevertheless has strong epistemic support, just because enough epistemic support can constitute justification.

I think we can resolve these conflicts by distinguishing the variable and threshold concepts of justification and distinguishing conditional justification from justification (which latter I shall call absolute justification when needing to draw particular attention to this distinction). There are correlate concepts of epistemic support. The degree of justification varies with the strength and extent of epistemic support. The conditionality of the epistemic support tracks the conditionality of justification. Completely unjustified beliefs are not epistemically supported by anything because if a belief has some epistemic support then it at least has some minimal degree of justification. It is possible for a belief to give strong conditional epistemic support to another even when the second lacks absolute justification. And so on.

My suggestion is that we have to disambiguate the definition of epistemic support and insist that the correlate concepts of epistemic support and justification be used on both sides of the biconditional. Doing so makes it clear that Stoneham could reasonably insist that (1) characterizes coherentism in either of two ways: when epistemic support is defined in terms of threshold justification; alternatively, when epistemic support is defined in terms of absolute justification, when the correlate concept of epistemic support is absolute, not conditional.

With these thoughts in mind I think we can see why Rowbottom's examples appeal and why they fail. They appeal because we take the

4 Stoneham's definition also mentions reasonableness. Since what I shall say about justifiedness can be said equally of reasonableness I shall not discuss reasonableness explicitly. Alternatively, we could strike 'reasonableness' from the definition. 
coherence got from the entailments and inductive support to provide a weak degree of justification and so we grant that those entailments and inductive support amount to epistemic support. Because entailment is a strong logical relation we take the presence of epistemic support to be undeniable. They fail either because the beliefs are threshold unjustified (thus undermining premiss A) or because foundationalism and coherentism do not agree on the cases (undermining premiss $\mathrm{B}$ ) or because the epistemic support is conditional (either undermining premiss A or making the step to $\mathrm{C}$ invalid by equivocation). I now spell this out.

\section{Under the threshold concept of justification}

Rowbottom's stipulation that the beliefs 'are not justified in an internal or external sense, except in so far as they might cohere' (2007: 345) is not innocent. It is necessary for the examples to satisfy the 'nothing else' clause of (1). Under foundationalism, however, the mere coherence of a belief with ungrounded or unjustified beliefs cannot result in the belief passing the threshold for justification.

In Rowbottom's trio, the support offered for the belief that all unicorns are white is inductive support given by the belief that there is a white unicorn. Under coherentism, it is not justified for two reasons. The first is that in doesn't pass the threshold for coherence by enumerative induction. Secondly, most varieties of coherentism apply some maximality condition on the set of beliefs with which a belief coheres, and three beliefs is just too small a number for any of the set to be coherence justified (a point which applies to all his examples).

So whether coherentism or foundationalism is true, none of the beliefs in his examples are threshold justified, and so there is no epistemic support between them when it is defined in terms of threshold justification.

It is tempting to think that this could be got round by a strengthened example, such as by adding the belief that every member of a non-empty representative sample of unicorns is white. ${ }^{5}$ If that belief is to get us past the threshold for justification it may also be necessary to add beliefs about the epistemic significance of such samples for inductive inference, or more general beliefs about methodology and the norms of inductive inference. Alternatively, it might be thought that I've loaded the example against Rowbottom by picking on such a normatively weighty belief needed only by internalists, and that the better strengthening in his aid is to supply the subject with lots of $1^{\text {st }}$ order beliefs that might suffice for externalist inductive justification. We should give him a hundred or a thousand or a

${ }^{5}$ My thanks to Michael Clark for raising this possibility. 
million beliefs with the contents ' $a$ is a white unicorn', ' $b$ is a white unicorn', and so on.

In either of these cases we have a proposed base for an inductive justification that all unicorns are white. Does the justification pass the threshold? Usually this kind of induction passes the threshold only in the context of or against a background of a great many other beliefs, and it is in such contexts that we usually make such inferences. So a coherentist can plausibly say that the inductive justification is absent otherwise. But suppose a coherentist must grant justification in these cases. It remains the case that a foundationalist cannot, for the reason already given - for him coherence alone does not justify, but coherence is all that is had here. So now foundationalism and coherentism do not agree on the cases.

\section{Under the variable concept of justification}

Suppose that in characterizing coherence (1) uses only the variable concept of justification. The case is not going to run as it stands under internalism. Our subject has only these three beliefs. Without additional beliefs in the entailments and inductive support the beliefs do not provide any degree of justification for each other. Externalists would be equally unhappy because the beliefs depend on the brainwashing rather than each other and reliable belief causing mechanisms. Dependence is also important in another respect, namely, we might think that a possessed belief being part of a potential justification for another is insufficient for epistemic support, which requires the basing relation to be standing between the beliefs. Now perhaps the internalist can stipulate knowledge of logic and the externalist some clever brainwashing process which is a reliable belief-causing mechanism such that each belief would not be had were the other two not had. And perhaps both can stipulate ways in which the basing relation holds between the beliefs. So suppose we set these problems aside. There is a further reason that the cases fail.

The coherence between the beliefs to which Rowbottom is appealing plays out differently for coherentists and foundationalists. Suppose a coherentist must concede that they are to some small degree justified, and so offer each other some degree of absolute epistemic support. A foundationalist may not. For him, whilst the coherence constitutes some degree of conditional justification, since there are no basic beliefs with some degree of justification for that conditional justification to transmit to those beliefs, the beliefs remain without any degree of justification. Hence by my insistence that the correlate concepts of justification and epistemic support appear in the definition, the beliefs provide no absolute epistemic support for one another. So once again, foundationalism and coherentism disagree on the cases. 
What about the conditional support? It is my contention that conditional support, however strong, is not a sort of support, but merely a promise of support. A beam in contact with the upper end of an immensely strong pillar which is not itself supported receives no support from the pillar, although it is true that if the pillar were supported then it would support the beam. Consequently, in taking (1) to characterize coherence, Stoneham could, and I think should, insist that the epistemic support is absolute, not conditional.

\section{Derivation of (1) from coherentism}

So there are two ways in which epistemic support and justification could be related without Stoneham's characterization of coherentism being true independently of coherentism. Furthermore, even if (1) is not adequate as a definition of coherentism, it is an entailment of a fairly standard kind of definition, together with the assumption that someone has got some justified beliefs. Hence the truth of coherentism can rise or fall with the truth of (1). Coherentism is the view that

(i) only beliefs can justify a belief and

(ii) justification of a belief is coherence with a justifying coherent set of beliefs to which the belief belongs.

What a justifying coherent set is depends on the theory of coherence and whether we are speaking of the variable concept, when perhaps any coherent set might count, or the threshold concept, when size and perhaps content requirements may be in force. ${ }^{6}$ Then we have the version of Stoneham's definition of epistemic support which I recommend:

(iii) One belief epistemically supports another belief just in case the latter is justified and its justification consists in part on its dependence on the former.

Throughout our premisses, we read 'justified' and 'justification' in terms of threshold justification or in terms of variable justification that is absolute, giving us the correlate variety of epistemic support throughout.

(iv) Someone has got some justified beliefs.

(v) Therefore '(1): There are finite sets of beliefs such that each member of the set is epistemically supported by some other members (and nothing else)'.

${ }^{6}$ We include varieties of coherentism in which the degree of coherence of a belief is simply the coherence of the set of which it is a member, when all beliefs have the same degree of coherence, and also varieties in which the degree of coherence of beliefs in a coherent set can vary from belief to belief, depending both on the degree of coherence of the set and on the nature and strength of their coherence with the set. 


\section{Proof}

We now proceed to the proofs that epistemic support is not asymmetric and of the equivalence for holistic coherentism. First we need some definitions. A directed graph is an ordered pair of a set of vertices and a set of directed edges, which latter are ordered pairs of members of the set of vertices. ${ }^{7}$ A visual representation of a directed graph can be given by points representing the vertices and arrows representing the directed edges. So for example, if Vertices $=\{a, b, c, d\}$ and Directed Edges $=\{\langle a, b\rangle,\langle b, c\rangle,\langle c, a\rangle$, $\langle b, d\rangle\}$, then we could represent this by:

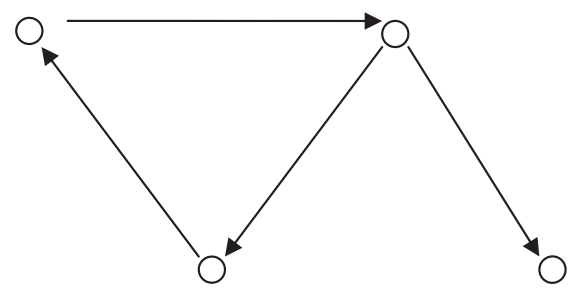

A directed cycle is a polygon of vertices joined by directed edges all pointing in the same direction round the polygon (so our example contains a directed cycle). A cyclic directed graph contains at least one directed cycle.

Lemma: If every vertex in a finite directed graph has an incoming edge then the graph is directed cyclic.

Proof: Suppose every vertex in a finite directed graph has an incoming edge. ${ }^{8}$ Starting with any vertex we can take a 'backwards' walk along the directed edges of as many steps as we please. Since there are only finitely many vertices, after at most as many steps as there are vertices in the graph, we must eventually return to a vertex we have already visited on the walk. The path we walked backwards between the first and second visit to that vertex is a directed cycle. Q.E.D.

Let $S$ be a set of beliefs, ${ }^{9} E$ be the relation of epistemic support and $R$ the extension of $E$ restricted to $S$. Then $\langle S, R\rangle$ is a directed graph, which we shall call the epistemic support graph of $S$, in which $S$ is the set of vertices and $R$ the set of directed edges. $R$ is a set of ordered pairs of beliefs in $S$, where an ordered pair is in $R$ just in case the first belief epistemically supports the second.

\footnotetext{
${ }^{7}$ For definitions of paths, walks, connectedness, completeness, cycles, indegrees, etc. see, for example, Bollobas 1998.

8 The indegree of every vertex $\geq 1$.

${ }^{9}$ Belief tokens, not types.
} 
Theorem 1: '(3) The relation of epistemic support is not asymmetric.' (Stoneham 2007: 255).

Proof: We have as premisses (1) and the quasi-transitivity of epistemic support. ${ }^{10}$ Let $S$ be one of the sets of beliefs mentioned in (1). $S$ is a finite set and so $\langle S, R\rangle$ is a finite directed graph. (1) implies that every member of $S$ is epistemically supported by some other member of $S$; consequently (since $R$ is the extension of epistemic support restricted to $S$ ) every vertex in $\langle S, R\rangle$ has an incoming edge in $R$ and so by the lemma $\langle S, R\rangle$ is a cyclic directed graph.

Now consider any distinct pair of vertices, $\{x, y\}$, in any directed cycle in $\langle S, R\rangle$. There is a finite directed path in that cycle from $x$ to $y$ and another from $y$ to $x$. The quasi-transitivity of epistemic support implies the quasi-transitivity of $R$. So by quasi-transitivity, there is a directed edge in $R$ from $x$ to $y$ and a directed edge in $R$ from $y$ to $x$. Since $R$ is the extension of $E$ restricted to $S,\{x, y\}$ is a pair of mutually supporting beliefs, and so epistemic support is not asymmetric. Q.E.D.

Theorem 2: Coherentist justification is holistic if and only if epistemic support is quasi-transitive and symmetric and the epistemic support graph of any set of justified beliefs of a person is connected. ${ }^{11}$

Proof: Left to Right: Coherentist justification being holistic means that the justification of a belief depends in part on all the beliefs in the justifying coherent set to which it belongs. So every belief in a justifying coherent set epistemically supports and is epistemically supported by all the other beliefs. So any pair of beliefs in a justifying coherent set are mutually supporting and hence epistemic support is symmetric and quasi-transitive. It also follows that every ordered pair of beliefs in a justifying coherent set belongs to the set of directed edges of its epistemic support graph, which is consequently complete. As a result, since a set of justified beliefs of a person is a subset of a justifying coherent set, the epistemic support graph of a set of justified beliefs must be connected.

Right to Left: Consider any justifying coherent set of beliefs, JCB, the subset, J, of all justified beliefs in JCB, and the subset, U, of beliefs that play some part in justifying at least one member of $\mathrm{J}$. If $\mathrm{U}$ is a proper subset of JCB then there are beliefs in JCB which play no part whatsoever in the justification of any belief in JCB. But that entails that they have no coherence whatsoever with any belief in JCB, which is to say they belong to JCB but are incoherent with JCB, contradicting its coherence. Hence $\mathrm{U}=\mathrm{JCB}$. By the definition of $\mathrm{U}$, each belief in JCB $\backslash \mathrm{J}$ epistemically supports

10 ‘(2*) $\forall x \forall y \forall z(x \neq z \wedge L x y \wedge L y z \rightarrow L x z)$ ' (Stoneham 2007: 255).

${ }^{11}$ Note also that if epistemic support is quasi-transitive and symmetric then an epistemic support graph is connected if and only if it is complete. 


\title{
234 ALEXANDER MILLER
}

at least one belief in $\mathrm{J}$ (vacuously true if $\mathrm{JCB} \backslash \mathrm{J}=\varnothing$ ). So in conjunction with the hypothesis that the epistemic support graph of $\mathrm{J}$ is connected it follows that the epistemic support graph of JCB is connected. Also by hypothesis epistemic support is symmetric and quasi-transitive. Consequently, every member of JCB epistemically supports and is epistemically supported by all the other beliefs. Hence the justification of every member of $J$ depends in part on all the beliefs in the justifying coherent set that it belongs to, through which belonging it is justified, and so coherentist justification is holistic. Q.E.D.

\author{
Cardiff University \\ Cardiff CF10 3EU, UK \\ shackeln@cardiff.ac.uk
}

\section{References}

Bollobas, B. 1998. Modern Graph Theory. New York: Springer.

Dretske, F. I. 1970. Epistemic operators. Journal of Philosophy 67: 1007-23.

Jäger, C. 2007. Is coherentism coherent? Analysis 67: 341-44.

Noordhof, P. 2007. A coherentist response to Stoneham's reductio. Analysis 67: 267-68.

Rowbottom, D. P. 2007. A refutation of foundationalism. Analysis 67: 345-46.

Silins, N. 2005. Transmission failure failure. Philosophical Studies 126: 71-102.

Stoneham, T. 2007. A reductio of coherentism. Analysis 67: 254-57.

Thagard, P. 2000. Coherence in Thought and Action. Cambridge, Mass.: MIT Press. 